\title{
Acellular pertussis vaccines
}

Twenty years ago, the whole cell pertussis vaccine used in Japan was withdrawn after the sudden death of two recently vaccinated infants. Although subsequently reinstated, concern about the risks of pertussis vaccination persisted, leading to poor uptake and a resurgence of disease. In response, the Japanese Ministry of Health launched a programme of research to develop a new vaccine containing purified pertussis antigens and devoid of active toxins which it was hoped would be more acceptable than whole cell vaccine. Within six years several new acellular pertussis vaccines with significantly lower reactogenicity than whole cell vaccine had been introduced in Japan, where they were reported to be successfully controlling whooping cough. ${ }^{1}$ However, the Japanese carried out no formal evaluation of vaccine efficacy in randomised controlled trials - a requirement for licensure in most other countries. Furthermore, in countries with successful whole cell vaccination programmes, relative efficacy data was considered essential before any decision could be made about replacement of the existing vaccine with acellular preparations. As no serological correlate of protection has been identified for pertussis, it was impossible to infer relative efficacy from antibody studies. An initial Swedish attempt to provide efficacy data for licensure from a placebo controlled trial was unsuccessful as efficacy estimates were disappointingly low and difficult to interpret in the absence of comparative data from a whole cell group. ${ }^{2}$

\section{Comparisons with whole cell vaccine}

Despite the enormous cost, renewed efforts were made by the American National Institute for Health and vaccine manufacturers to obtain relative efficacy data. By 1992, several large efficacy trials had been mounted in countries such as Sweden, Italy, and Germany where whole cell vaccine uptake was sufficiently low, and disease incidence sufficiently high, to allow a comparison of whooping cough attack rates in cohorts of whole cell and acellular vaccinees. None of the trials used the UK accelerated schedule of 2/3/4 months, most being conducted using the American schedule of $2 / 4 / 6$ months.

On 13 July 1995, the first results of the Swedish and Italian trials were announced (Swedish Institute for Infectious Disease Control press release). A total of four different acellular vaccines were evaluated and compared with an established American whole cell vaccine using a double blind randomised trial design and a 2/4/6 month schedule. All pertussis vaccines were evaluated as combined diphtheria, tetanus, pertussis (DTP) vaccines with the control group receiving diphtheria and tetanus vaccine. The four acellular DTP vaccines all contained filamentous haemagglutinin (FHA) - a surface antigen probably involved in protection against colonisation, and pertussis toxin - the major systemic toxin of the organism ${ }^{3}$ The pertussis toxin component of one of the four vaccines was derived from a mutated Bordetella pertussis strain which produces a non-toxic form of pertussis toxin. Avoidance of chemical toxoiding with agents such as formaldehyde, which is the conventional way of removing toxic activity, preserves the tertiary structure of the pertussis toxin molecule and should result in a more effective immunogen. ${ }^{4}$ In addition to FHA and pertussis toxin, three of the vaccines also contained an outer membrane protein (pertactin) which had been shown to be protective in an animal model. ${ }^{3}$ One vaccine also contained serotype specific agglutinogens 2 and 3 for which indirect evidence of protection has been obtained from both epidemiological studies and animal models. 56

The results were rather surprising. Despite epidemiological evidence from the USA showing evidence of high efficacy ${ }^{7}$ the American whole cell DTP vaccine gave only $36 \%$ protection (95\% confidence interval (CI) 14 to $52 \%$ ) in the Italian trial and $48 \%(95 \%$ CI 37 to $57 \%)$ in the Swedish trial. It is possible that the fourth and fifth doses of whole cell vaccine given in the USA at 18 months and at school entry compensate for the low efficacy of the American vaccine. In contrast the three and four component acellular DTP vaccines gave $84 \%$ protection after the three dose course (95\% CI 76 to $90 \%)$. Although the vaccine containing the genetically detoxified pertussis toxin did not provide better protection, equivalent protection was achieved with one fifth of the dose. This superior immunogenicity may prove to be a significant advantage when the acellular DTP vaccines are mixed with additional vaccine antigens. The two component pertussis toxin/FHA vaccine gave only $58 \%$ protection ( $95 \%$ CI 50 to $65 \%$ ) confirming that immunity to several pertussis components is necessary to achieve satisfactory protection. The hypothesis that pertussis, like diphtheria and tetanus, is essentially a toxin mediated disease ${ }^{8}$ has been finally laid to rest. Although the duration of protection was not studied in the trials it is likely to be similar to the protection provided by immunisation with whole cell vaccine.

The pertussis case definition used for the primary analysis was paroxysmal cough of at least 21 days' duration with laboratory evidence of pertussis infection. The latter criterion required isolation of $B$ pertussis, or a significant rise in antibodies to pertussis toxin or FHA ( $45 \%$ of the total confirmed cases), or identification of $B$ pertussis organisms by the polymerase chain reaction. In practice, the polymerase chain reaction contributed few additional cases, as swabbing and serological investigation of suspected cases was instituted early. However, the potential value of this method in cases investigated relatively late in the illness may be greater. Because of the lower sensitivity of culture and serological methods in confirming cases in vaccinated than unvaccinated children, ${ }^{9}$ secondary analyses based on clinical case definitions will be carried out which may give lower absolute efficacy estimates.

For the UK, however, the main outcome of interest will be the results of a second Swedish trial, due to end in 1996, in which the three and four component acellular DTP vaccines are being compared with UK whole cell DTP vaccine made by Wellcome. This trial was designed to provide precise relative efficacy estimates and is substantially larger than the first (approximately 20000 per vaccine group compared with 2500 ). The Swedish primary immunisation schedule of $3 / 5 / 12$ months is being used. The efficacy estimates for the Wellcome DTP vaccine will be of considerable interest in the UK. Pertussis surveillance data from England and Wales suggest a highly effective vaccine, ${ }^{10}$ with studies after licensure giving estimates of around $90 \%$ for protection against clinically typical or bacteriologically confirmed cases. ${ }^{11} 12$ With the current vaccine coverage of over $90 \%$, which has now been sustained for a number of years, disease incidence is at an all time low. Furthermore, there is convincing evidence that the Wellcome whole cell vaccine induces herd immunity as there has been a progressive decline in cases under 2 months of age - a group who are too young to be protected 
directly by vaccination. ${ }^{13}$ A major re-evaluation of our pertussis surveillance systems will therefore be necessary if efficacy estimates as low as those obtained for American whole cell vaccine emerge for the UK whole cell vaccine from the ongoing Swedish trial.

The Swedish and Italian trials provided unequivocal evidence of the improved safety profile of the acellular DTP vaccines. As expected, common symptoms such as fever and irritability were significantly reduced and as a result, completion of the three dose course was $2-3 \%$ higher in the acellular than whole cell groups. The more alarming reaction of hypotonic hyporesponsive episodes occurred almost exclusively in the whole cell group at a rate of about one in 1000 doses, primarily the first. Although not resulting in sequelae, these episodes are frightening for parents and may represent a mild form of endotoxic shock, associated with the higher levels of endotoxin in whole cell than acellular vaccines.

\section{Prospects for routine use}

Assuming that the second Swedish trial shows equivalent efficacy for UK whole cell and the acellular DTP vaccines, what are the implications? For countries such as the UK, where acceptance of whole cell vaccine is high, further improvements in coverage by switching to acellular preparations are likely to be limited to the few per cent of children who fail to complete because of post-vaccination symptoms contraindicating further doses. This by itself is unlikely to justify the extra cost of these new vaccines. For developing countries the extra cost of the vaccine may not be justified because problems with achieving wide coverage are of paramount importance. However, a reduction in reactogenicity may become a more significant advantage when new combination vaccines are introduced. The Children's Vaccine Initiative (a collaborative venture involving international organisations, industry, research and public health agencies) has identified DTP vaccine as the platform on which to build future combination vaccines for paediatric use, particularly those incorporating components which provide protection against bacterial meningitis. Acellular pertussis components are likely to be better than whole $B$ pertussis organisms for inclusion in combination vaccines as they are superior immunogens and no more reactogenic than existing diphtheria and tetanus vaccines. The highly purified nature of acellular pertussis vaccines is also an advantage for regulatory agencies as the pharmaceutical properties of the vaccine can be chemically defined.

It is envisaged that acellular pertussis vaccines, when combined with other vaccines, will have wide application in primary immunisation schedules for infants and for boosting immunity in older age groups. Although no booster is currently given in the $\mathrm{UK}$, it is likely that inclusion of pertussis vaccine in the preschool booster will be recommended as there is now little opportunity for natural boosting by exposure to whooping cough. The use of whole cell vaccine for boosting is probably unacceptable as there is a substantial increase in reactogenicity when using DTP compared with diphtheria and tetanus vaccine as the preschool booster. ${ }^{14}$ Furthermore, recent experience in the USA, where there has been a recent resurgence of pertussis in adults, suggests that an extra dose at school leaving age may be necessary to ensure continuing protection in adulthood. ${ }^{15}$
In anticipation of these developments, a three year clinical trials programme sponsored by the Department of Health and carried out by the Public Health Laboratory Service (PHLS) has begun. The aim is to provide a scientifically independent evaluation of the various acellular vaccines now available and their suitability for use in the UK population. The information will allow the performance of the different vaccines under the $2 / 3 / 4$ month schedule to be compared with each other and with the existing UK whole cell vaccine with respect to reactogenicity and immunogenicity. Their compatibility when given as a single injection mixed with other paediatric vaccines such as Haemophilus influenzae type b, hepatitis $B$, meningococcal, or inactivated poliovirus vaccines will also be assessed. Three acellular DTP vaccines have already been evaluated in UK infants at 2,3, and 4 months of age and found to be highly immunogenic and, unlike whole cell vaccine, not inhibited in the presence of high levels of maternal antibody (PHLS, unpublished). Follow up of the children who have been vaccinated in the primary immunisation trials will provide antibody persistence data in advance of the introduction of a national programme and will allow the need for booster doses to be assessed. The acceptability of acellular DTP vaccines for preschool boosting will also be evaluated and the use of acellular vaccines combined with tetanus and low dose diphtheria vaccines will be investigated in adolescents.

It is intended that the acellular pertussis clinical trials programme will serve as a model for the evaluation of other paediatric vaccines, such as pneumococcal conjugates and rotavirus vaccines, which are likely to become available in the UK and Europe in the near future.

ELIZABETH MILLER

\section{Immunisation Division,}

Public Health Laboratory Service,

Communicable Disease Surveillance Centre,

61 Colindale Avenue,

London NW9 $5 E Q$

1 Kimura M, Kuno-Sakai H. Developments in pertussis immunisation in Japan. Lancet 1990; 336: 30-2.

2 Ad Hoc Group for the Study of Pertussis Vaccines. Placebo controlled trial of two acellular pertussis vaccines in Sweden - protective efficacy and adverse events. Lancet 1988; i: 955-60.

3 Robinson A, Ashworth LAE. Acellular and defined-component vaccines against pertussis. In: Wardlaw AC, Parton R, eds. Pathogenesis and against pertussis. In: Wardlaw AC, Parton R, eds. Path
immunity in pertussis. Chichester: John Wiley 1988: 399-417.

4 Rappuoli R, Pizza M, Covacci A, et al. Recombinant acellular pertussis vaccine from the laboratory to the clinic: improving the quality of the vaccine from the laboratory to the clinic: improving the quality
immune response. FEMS Microbiol Immunol 1992; 105: 161-70.

5 Preston NW, Cater EJ. Serotype specificity of vaccine-induced immunity. Communicable Disease Report Review 1992; 2: R155-66.

6 Robinson A, Gorringe AR, Funnell SGP, Fernandez M. Serospecific protection of mice against intranasal infection with Bordetella pertussis. Vaccine 1989; 7: 321-4.

7 Onorato IM, Wassilak SG, Meade B. Efficacy of whole-cell pertussis vaccine in pre-school children in the United States. $\mathcal{F} A M A$ 1992; 267: 2745-9.

8 Pittman M. The concept of pertussis as a toxin-mediated disease. Pediatr Infect Dis ₹ 1984; 3: 467-86.

9 Storsaeter J, Hallander H, Farrington CP, Olin P, Mollby R, Miller E. Secondary analyses of the efficacy of two acellular pertussis vaccines in a Swedish phase III trial. Vaccine 1990; 8: 457-61.

10 Miller E, Vurdien JE, White JM. The epidemiology of pertussis in England and Wales. Communicable Disease Report Review 1992; 2: R152-4.

11 PHLS Epidemiological Research Laboratory and 21 Area Health Laboratories. Efficacy of pertussis vaccination in England. $B M F$ 1982; 285: 357-9.

12 Ramsay M, Farrington P, Miller E. Age-specific efficacy of pertussis vaccine during epidemic and non-epidemic periods. Epidemiol Infect 1993; 111: 41-8

13 Miller E, White JM, Fairley CK. Pertussis vaccination [Letter]. Lancet 1994; 344: 1575-6.

14 Miller E, Rush M, Ashworth LAE, et al. Antibody responses and reactions to the whole cell pertussis component of a combined diphtheria/tetanus/pertussis vaccine given at school entry. Vaccine (in press).

15 Edwards KM. Acellular pertussis vaccines - a solution to the pertussis problem? $\mathcal{F}$ Infect Dis 1993; 168: 15-20. 